\title{
Phase-coherent all-optical frequency division by three
}

\author{
Dong-Hoon Lee, ${ }^{*}$ Marvin E. Klein ${ }^{\dagger}$ Jan-Peter Meyn, and Richard Wallenstein \\ Fachbereich Physik, Universität Kaiserslautern, Erwin-Schrödinger-Straße 46, 67663 Kaiserslautern, Germany \\ Petra Groß $\aleph^{\ddagger}$ and Klaus-Jochen Boller \\ Faculty of Applied Physics, University of Twente, P. O. Box 217, 7500 AE Enschede, The Netherlands
}

(Received 4 September 2002; published 23 January 2003)

\begin{abstract}
The properties of all-optical phase-coherent frequency division by 3, based on a self-phase-locked continuous-wave (cw) optical parametric oscillator (OPO), are investigated theoretically and experimentally. The frequency to be divided is provided by a diode laser master-oscillator power-amplifier system operated at a wavelength of $812 \mathrm{~nm}$ and used as the pump source of the OPO. Optical self-phase-locking of the OPO signal and idler waves is achieved by mutual injection locking of the signal wave and the intracavity frequency-doubled idler wave. The OPO process and the second-harmonic generation of the idler wave are simultaneously phase matched through quasi-phase-matching using two periodically poled sections of different period manufactured within the same $\mathrm{LiNbO}_{3}$ crystal. An optical self-phase-locking range of up to $1 \mathrm{MHz}$ is experimentally observed. The phase coherence of frequency division by three is measured via the phase stability of an interference pattern formed by the input and output waves of the OPO. The fractional frequency instability of the divider is measured to be smaller than $7.6 \times 10^{-14}$ for a measurement time of $10 \mathrm{~s}$ (resolution limited). The self-phase-locking characteristics of the cw OPO are theoretically investigated by analytically solving the coupled field equations in the steady-state regime. For the experimental parameters of the OPO, the calculations predict a locking range of $1.3 \mathrm{MHz}$ and a fractional frequency instability of $1.6 \times 10^{-15}$, in good agreement with the experimental results.
\end{abstract}

DOI: 10.1103/PhysRevA.67.013808

PACS number(s): 42.62.Eh, 42.65.Yj, 42.79.Nv, 42.65.Ky

\section{INTRODUCTION}

Continuous-wave (cw) optical parametric oscillators (OPOs) are widely tunable sources of coherent light in the near and mid infrared. In addition to their importance for applications in high-resolution spectroscopy, cw OPOs are also promising candidates for wavelength-flexible, phasecoherent, all-optical frequency dividers and synthesizers for high-precision optical metrology, due to their high efficiency, high resolution, and high precision [1].

Recently, a phase-coherent link between the current time standard (microwave atomic clock based on Cs) and optical frequencies in the visible and near-infrared range has been realized by using a cw mode-locked laser followed by wideband optical comb generation in a nonlinear fiber [2]. Nevertheless, in order to measure with the same precision any unknown optical frequency lying further in the infrared than the frequency comb (i.e., beyond $1.5 \mu \mathrm{m}$ in the mid infrared), subsequent steps of phase-coherent frequency division are required. A promising candidate for this task is all-optical division by the integer number 2 or 3 using a cw OPO. Such phase-coherent division of a visible or near-infrared frequency from a comb or a laser can be realized by an OPO with the mid-infrared idler frequency tuned to precisely onehalf or one-third of the pump laser frequency.

\footnotetext{
*Present address: KAIST, Center for Electro-Optics, GuseongDong, Yuseong-Gu, 305-701 Daejeon, Republic of Korea.

†Present address: Nederlands Centrum voor Laser Research, NCLR b.v., P. O. Box 2662, 7500 CR Enschede, The Netherlands.

${ }^{\ddagger}$ Corresponding author. Email address: p.gross@tn.utwente.nl
}

The precision of such frequency division is characterized by the divider's stability, i.e., the deviation of the idler frequency from the exactly divided pump frequency. While the phase sum $\phi_{S}+\phi_{I}$ of the idler and signal waves adiabatically follows the pump phase, the phase difference $\phi_{S}-\phi_{I}$ undergoes a diffusion process [3]. In order to achieve phasecoherent frequency division, this diffusion has to be suppressed by some phase-locking mechanism. The residual variance of the phase difference then determines the divider's stability. The required phase coherence of the idler with respect to the pump field oscillation (and thus of the division by an integer number) can be achieved either by an electronic or by an optical phase control of the OPO fields.

In the case of an electronic control, the frequency and phase difference between the signal and idler waves needs first to be determined via a beat measurement which generates a rf error signal, if the signal and idler frequencies are sufficiently close to each other. The electronic error signal can then be used to control the OPO frequencies and phases via a tuning element in the OPO setup, e.g., a piezoelectric transducer, which controls the OPO cavity length. For a division by 2 , based on an OPO tuned close to degeneracy, a rf error signal can readily be achieved by directly beating the signal with the idler wave [4]. Electronically controlled division by 3 is more involved, because the idler wave needs first to be frequency doubled before a beat with the signal wave can be measured in the $\mathrm{rf}$ range $[5,6]$.

Another problem with electronically phase-locked division by 3 is a slow response time, which limits the stability of the divider. The optical power available after externally frequency-doubling the OPO idler output wave is low, typically in the nanowatt range, such that the rf error signal 
needs to be averaged over milliseconds to seconds for a sufficiently high signal-to-noise ratio in the feedback loop. Another limitation could be the response time of the piezoelectric transducer, which also lies on the order of milliseconds.

An all-optical phase locking does not suffer from these restrictions. It rather offers a response as fast as the inverse bandwidth of the OPO's cold cavity modes, which is on the order of tens of nanoseconds.

To control the phases of an OPO divider with all-optical methods, one of the output waves is phase locked by injecting another optical wave, similar to injection locking of lasers [7]. If the injected wave is generated by the OPO itself, the method is termed self-phase-locking. The most obvious example for such self-phase-locking is a frequencydegenerate, type-I phase-matched $\mathrm{cw}$ OPO, where the signal and idler wave polarizations are parallel, such that the signal wave phase-locks the idler wave and vice versa [8]. Frequency division by 2 can also be achieved with a type-II phase-matched cw OPO (with orthogonal signal and idler polarization) by introducing a wave plate in the OPO cavity [9].

All-optical division by 3 was recently demonstrated in our previous work with a cw OPO, where the signal wave is injection locked through the intracavity frequency-doubled idler wave [10,11]. In Ref. [12], a theoretical model was developed and numerically evaluated to analyze its steady state and dynamics. Here, we use a different approach to analytically predict the self-phase-locking behavior of our divider OPO, and we compare the predicted locking range and frequency stability with experimental results. We measured an optical locking range of the OPO between 0.5 and 1 $\mathrm{MHz}$, depending on the setup, and we measured a fractional frequency instability of the division of smaller than 7.6 $\times 10^{-14}$ for a measurement time of $10 \mathrm{~s}$ (resolution limited). These experimental results agree well with the theoretically predicted values.

In the next section, we derive analytical expressions for the locking range and the frequency stability of the selfphase-locked OPO by solving the coupled field equations in the steady-state regime. In Sec. III, we describe the experimental setup to measure the locking range and stability of the frequency divider, and we discuss the results obtained with this setup.

\section{THEORETICAL ANALYSIS}

The basic concept for optical self-phase-locking is injection locking [7] of a cw OPO by an optical wave whose frequency is close to the signal or idler frequency. For the present example of optically self-phase-locked division by 3 , we use a cw OPO, where intracavity frequency doubling [second-harmonic generation (SHG)] of the idler wave generates the injected field to phase-lock the signal wave of the OPO. The schematic setup of the experiment is shown in the upper part of Fig. 1, while the lower part shows a corresponding frequency diagram of the nonlinear processes involved.

The OPO consists of an optically nonlinear crystal in a linear two-mirror cavity. The cavity is assumed to be reso-
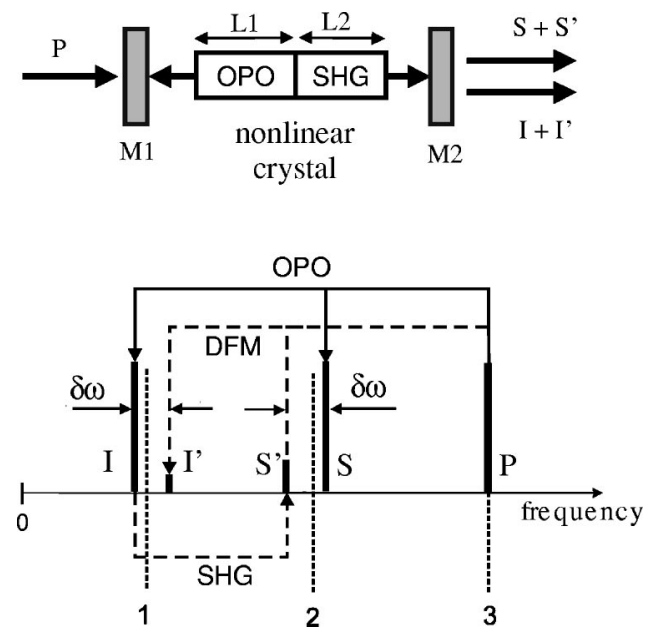

FIG. 1. Scheme for an OPO with intracavity idler SHG for frequency division by 3 (upper part, M1, M2 are cavity mirrors), and frequency diagram of the involved nonlinear processes (lower part). By SHG of the idler wave $I$, the wave $S^{\prime}$ is generated, which is separated from the signal wave $S$ by $\delta \omega$. At the same time, via DFM of the doubled idler wave with the pump wave, a field $\left(I^{\prime}\right)$ is generated that deviates from $I$ again by $\delta \omega$. For further details, refer to the text.

nant for the pump $(P)$, signal $(S)$, and idler $(I)$ waves. We assume that the crystal simultaneously provides phase matching for the OPO process and for SHG of the idler wave. Further details on such a crystal will be described in Sec. III. Via the OPO process, the pump frequency $\omega_{P}$ is divided into a signal frequency $\omega_{S}$ and an idler frequency $\omega_{I}$ with the ratio $\omega_{P}: \omega_{S}: \omega_{I}$ of approximately $3: 2: 1$. By SHG of the idler wave, an additional wave $\left(S^{\prime}\right)$ at the frequency $2 \omega_{I}$ is generated, which is close to the signal frequency $\omega_{S}$. The difference between the two light frequencies is defined as $\delta \omega:=\omega_{S}-2 \omega_{I}$. Due to phase matching of the idler SHG, difference-frequency mixing (DFM) of the doubled idler wave $S^{\prime}$ with the pump wave should enable the generation of a field with a frequency $\omega_{P}-2 \omega_{I}\left(\right.$ labeled $\left.I^{\prime}\right)$, which deviates from $\omega_{I}$ again by $\delta \omega$. For an overview, these nonlinear processes are indicated in the lower part of Fig. 1 by arrows.

If the frequency difference $\delta \omega$ is adjusted by tuning the phase-matching wavelengths and the cavity length of the $\mathrm{OPO}$, such that $\delta \omega$ becomes smaller than a characteristic frequency difference $\delta \omega_{\text {lock }}$, i.e., $|\delta \omega| \leqslant \delta \omega_{\text {lock }}$, one expects injection locking of the signal and idler waves by the waves $S^{\prime}$ and $I^{\prime}$, respectively. The full interval of $2 \delta \omega_{\text {lock }}$ within which injection locking occurs is called the locking range. This injection locking of the OPO waves is analogous to the well-known injection locking of lasers [7]. In a laser injection locking occurs if the frequencies of the injected wave and the free-running wave of an oscillator lie within the locking range. In this case the free-running wave assumes precisely the frequency of the injected wave. The same should occur in an OPO, with the difference that, in the considered OPO injection locking, both waves are generated by the same process, namely, optical parametric oscillation. This situation can thus be termed self-injection-locking. In the case that the signal and the idler waves are injection 
locked by the waves $S^{\prime}$ and $I^{\prime}$, the pump, signal, and idler waves should oscillate with the exact frequency ratio of $\omega_{P}: \omega_{S}: \omega_{I}=3: 2: 1$. The corresponding signal and idler frequencies are indicated in Fig. 1 by dashed lines.

If the free-running wave of a laser is injection locked, its phase is also determined with respect to the phase of the injected wave, i.e., both waves oscillate phase coherently. A similar effect should be observed for a self-injection-locked OPO: Without locking, the phases $\phi_{S}$ and $\phi_{I}$ of the signal and the idler waves are free with respect to each other. Only their sum is determined by the phase of the pump wave [3]. When the OPO waves are self-injection-locked, however, their phase difference $\phi_{S}-\phi_{I}$ is determined as well. Therefore, the three OPO waves oscillate in phase with each other, and the frequency division of the pump laser can be called phase coherent.

In the following, we start from the coupled wave equations of an OPO with intracavity idler SHG, to derive analytically the locking range of the OPO and to determine its theoretically expected frequency stability.

\section{A. Coupled field equations}

The nonlinear wave mixing processes taking place in the OPO can be summarized in the mean-field, plane-wave approximation by three coupled equations for the OPO internal electric field $q(t)=q_{P}(t) e^{i \Omega_{P} t}+q_{S}(t) e^{i \Omega_{S} t}+q_{I}(t) e^{i \Omega_{I^{t}}}$. The carrier frequencies $\Omega_{P}, \Omega_{S}$, and $\Omega_{I}$ are chosen to posses the exact ratio of $3: 2: 1$, respectively. The amplitudes $q_{P}, q_{S}$, and $q_{I}$ are slowly varying envelopes, which contain also the frequency deviation of the OPO fields from those carriers, i.e., they contain the frequency deviation of the OPO light fields from phase-coherent division by $3, \omega_{S}-\Omega_{S}$ and $\omega_{I}$ $-\Omega_{I}$. We chose $\Omega_{P} \equiv \omega_{P}$, such that the carrier frequencies $\Omega_{P}, \Omega_{S}$, and $\Omega_{I}$ are determined by the pump laser frequency $\omega_{P}$. The coupled equations for the field amplitudes can then be written as follows [13]:

$$
\begin{gathered}
\frac{d q_{P}}{d t}=-\frac{1}{2}\left(\kappa_{P}-i \Delta_{P}\right) q_{P}+i D q_{S} q_{I}+F, \\
\frac{d q_{S}}{d t}=-\frac{1}{2}\left(\kappa_{S}-i \Delta_{S}\right) q_{S}+i D q_{I}^{*} q_{P}+\frac{1}{2} i G q_{I} q_{I}, \\
\frac{d q_{I}}{d t}=-\frac{1}{2}\left(\kappa_{I}-i \Delta_{I}\right) q_{I}+i D q_{P} q_{S}^{*}+i G q_{S} q_{I}^{*} .
\end{gathered}
$$

The field amplitudes $q_{x}$ are normalized so that the squares of their absolute values give the intracavity photon numbers $N_{x}=\left|q_{x}\right|^{2}$, where the indices $x=P, S$, and $I$ designate the pump, signal, and idler waves, respectively. The cavity decay rates

$$
\kappa_{x}=\mathcal{F}_{x}\left|\ln \left[\left(1-T_{x 1}\right)\left(1-T_{x 2}\right)\left(1-A_{x}\right)^{2}\right]\right|
$$

are defined with the power transmissions $T_{x 1}$ and $T_{x 2}$ of the cavity mirrors $\mathrm{M} 1$ and $\mathrm{M} 2$, respectively (see Fig. 1), and the passive single-pass losses $A_{x} . \quad \mathcal{F}_{x}=(c / 2)\left[L_{\text {cav }}+\left(L_{1}\right.\right.$ $\left.\left.+L_{2}\right)\left(n_{x}-1\right)\right]$ are the free spectral ranges of the cavity with

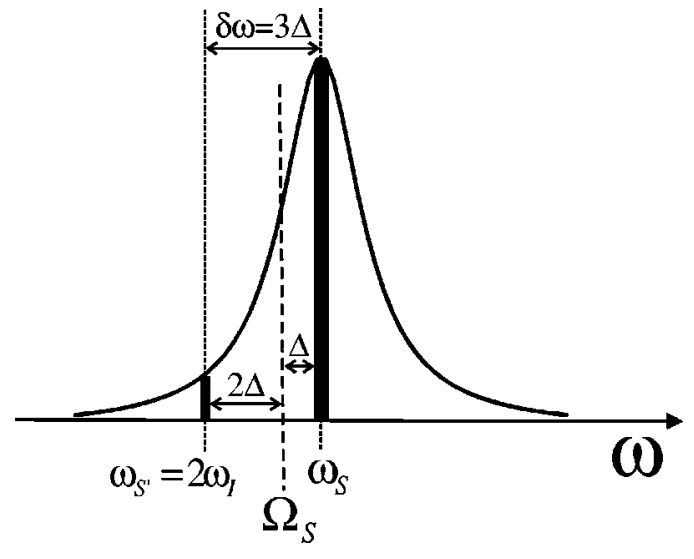

FIG. 2. Definition of the frequency detuning $\Delta$ (considered, e.g., for the signal wave). After a maximization of the output power via both cavity detunings $\Delta_{S}$ and $\Delta_{I}$, the signal frequency $\omega_{S}$ of the free-running OPO can be assumed to have zero detuning from the cold cavity resonance, symbolized by the Lorentz-shaped solid line. $\Delta=\omega_{S}-\Omega_{S}$ is the detuning of the signal frequency $\omega_{S}$ from the exact two-thirds of the pump frequency $\Omega_{S}=2 \Omega_{P} / 3$. The SH frequency of the idler wave can be calculated to be $\omega_{S^{\prime}}=2 \omega_{I}$ $=\Omega_{S}-2 \Delta$. The difference between the frequencies of the signal wave and the frequency-doubled idler wave thus becomes $\delta \omega$ $=\omega_{S}-\omega_{S^{\prime}}=3 \Delta$.

the geometrical cavity length $L_{\text {cav }}$, the geometrical crystal lengths $L_{1}$ and $L_{2}$ of the OPO crystal and the SHG crystal, respectively, and the refractive indices of the crystal $n_{x} . \quad \Delta_{x}$ are the detunings of the OPO's cold cavity modes from exact division by 3 . A zero detuning of the pump cavity from the pump frequency $\Delta_{P}=0$ is assumed because in the experiment the pump cavity length is electronically locked to the pump laser wavelength.

To investigate frequency division by 3 , we only consider the case that the value of $\delta \omega$ is so small that the fields $S$ and $S^{\prime}$ (and thus also $I$ and $I^{\prime}$ ) fall within the same mode of the cold cavity, so that injection locking can occur. In the experiment we tuned the pump frequency and the cavity length such that the free-running OPO (without measuring the idler SHG frequency) oscillated with maximum output power and thus minimum threshold. For this case we can assume that both the signal and idler waves oscillate at the cold cavity mode center frequencies [14]. The light frequencies $\omega_{S}$ and $\omega_{I}$ are detuned from the exact divider frequencies $\Omega_{S}$ and $\Omega_{I}$ by $\Delta_{S}$ and $\Delta_{I}$, respectively, i.e., $\omega_{S}=\Omega_{S}+\Delta_{S}$ and $\omega_{I}=\Omega_{I}$ $+\Delta_{I}$. From the two conditions of energy conservation, $\omega_{P}$ $=\omega_{S}+\omega_{I}$ and $\Omega_{P}=\Omega_{S}+\Omega_{I}$, and from $\omega_{P} \equiv \Omega_{P}$, it follows that the detunings $\Delta_{S}$ and $\Delta_{I}$ are of the same value, but opposite sign. For the power-optimized OPO we can thus replace the signal and idler cavity detuning parameters by a single parameter $\Delta$ with $\Delta:=\Delta_{S} \equiv-\Delta_{I}$. The detuning $\Delta$ corresponds to the deviation of the signal and idler light frequencies from the desired frequencies of the divider, which lie at precisely two-thirds and one-third of the pump frequency, respectively. This definition of the frequency detunings is depicted schematically in Fig. 2.

With this definition of $\Delta$, the earlier considered frequency difference between the signal wave $S$ and the idler SHG 
wave $S^{\prime}$ can be expressed in terms of $\Delta$ as well, i.e.,

$$
\delta \omega=\omega_{S}-\omega_{S^{\prime}}=\omega_{S}-2 \omega_{I}=3 \Delta .
$$

Consequently, the full locking range $2 \delta \omega_{\text {lock }}$ can be expressed as $2 \delta \omega_{\text {lock }}=6 \Delta_{\text {lock }}$. For cavity detunings smaller than the characteristic detuning $\Delta_{\text {lock }}$, i.e., $|\Delta| \leqslant \Delta_{\text {lock }}$, one expects injection locking of the signal and idler waves by the waves $S^{\prime}$ and $I^{\prime}$, respectively.

The pump rate $F$ is proportional to the square root of the external pump laser power $P_{P}$ times the pump input transmission $T_{P 1}$ of the cavity mirror M1: $F^{2}$ $=\mathcal{F}_{P} T_{P 1}\left(P_{P} / \hbar \omega_{P}\right)$. Assuming phase matching, the nonlinear coupling coefficient $D$ for the OPO process is defined as

$$
D=\frac{d_{\mathrm{eff}(1)}}{4 n_{P} n_{S} n_{I}} \sqrt{\left(2 \hbar / \varepsilon_{0} V_{1}\right) \omega_{P} \omega_{S} \omega_{I}}\left(\frac{L_{1}}{L_{\mathrm{cav}}}\right),
$$

where $d_{\text {eff(1) }}$ is the effective nonlinear coefficient, $V_{1}$ the volume of the nonlinear interaction, and $n_{x}$ the refractive indices.

The last terms on the right-hand side (RHS) of the second and third lines of Eqs. (1) describe the additional SHG of the idler wave $\frac{1}{2} i G q_{I} q_{I}$ and signal-idler difference-frequency mixing (DFM) $i G q_{S} q_{I}^{*}$. These processes contribute the frequency components $S^{\prime}$ and $I^{\prime}$ close to the signal and idler waves. In analogy to Eq. (4), the nonlinear coupling coefficient for the DFM process and for the idler SHG process is given by

$$
G=\frac{d_{\mathrm{eff}(2)}}{4 n_{S} n_{I}^{2}} \sqrt{\frac{2 \hbar}{\varepsilon_{0} V_{2}} \omega_{S} \omega_{I}^{2}}\left(\frac{L_{2}}{L_{\mathrm{cav}}}\right),
$$

with the effective nonlinear coefficient $d_{\mathrm{eff}(2)}$ and the volume of nonlinear interaction $V_{2}$ of the SHG crystal.

The coupled field equations can be solved for two very different regimes of oscillation. First, there is the regime of oscillation where the frequency difference between the signal wave and the idler SHG wave is larger than the locking range, i.e., $|\delta \omega|>\delta \omega_{\text {lock }}$ and, equivalently, $|\Delta|>\Delta_{\text {lock. In }}$ this case, the solutions for the field envelopes in Eqs. (1) will not be stationary, but a (periodic) function of time, which means that the frequency division by the OPO is not phase locked.

In the second regime of oscillation, where the detuning is within the locking range, i.e., $|\Delta| \leqslant \Delta_{\text {lock }}$, the solutions of Eqs. (1) will be time independent, indicating that the signal and idler waves of the OPO divider will be self-phaselocked, such that the OPO works as a phase-coherent frequency divider. This behavior has been observed in our previous numerical calculations [11], in reasonable agreement with experimental results. In the current work, the coupled field equations (1) are solved analytically in the steady-state regime, and corresponding expressions for the locking range, for the phase eigenvalues, and for the frequency stability of the self-phase-locked OPO are derived.

\section{B. Locking range}

In the following, the nonlinear coupled equations (1) are solved for the locking range, with the OPO cavity detuning $\Delta$ being a variable parameter, which can be independently adjusted in the experiment. The other construction parameters of the OPO, such as the cavity losses or the crystal's nonlinear coefficient, are fixed and can be derived from the experimental setup in independent measurements of, e.g., the OPO threshold pump power or the OPO output power.

For a simplification of the equations, we assume that the change of the steady-state field amplitude $q_{I}$ induced through the idler SHG and the signal-idler DFM processes can be neglected with regard to the gain of the OPO process. This approximation of weak idler SHG is well justified for our experimental setup, where we measured that the power ratio of the frequency-doubled idler wave and the signal wave is smaller than $-25 \mathrm{~dB}$. With weak idler SHG, we can regard the complex quantity $i G q_{I}$ on the RHS of the last two equations in (1) as being constant, and define $\varepsilon:=i G q_{I}$. This complex parameter $\varepsilon$ can be seen as an optical injection term between the signal and idler fields, similar to the one introduced by Fabre et al. to describe self-injection-locking of a type-II OPO by 2 divider [15]. Considering steady-state conditions [i.e. zero time derivatives on the LHS of Eqs. (1)], one obtains four linear coupled equations for $q_{S}$ and $q_{I}$ and their complex conjugates $q_{S}^{*}$ and $q_{I}^{*}$ :

$$
\begin{array}{ccccc}
\frac{1}{2}\left(\kappa_{S}+i \Delta\right) & -\frac{1}{2} \varepsilon & 0 & -i D q_{P} \\
\varepsilon^{*} & \frac{1}{2}\left(\kappa_{I}-i \Delta\right) & -i D q_{P} & 0 \\
0 & i D q_{P}^{*} & \frac{1}{2}\left(\kappa_{S}-i \Delta\right) & -\frac{1}{2} \varepsilon^{*} \\
i D q_{P}^{*} & 0 & \varepsilon & \frac{1}{2}\left(\kappa_{I}+i \Delta\right) \\
\times\left(\begin{array}{c}
q_{S} \\
q_{I} \\
q_{S}^{*} \\
q_{I}^{*}
\end{array}\right)=\left(\begin{array}{c}
0 \\
0 \\
0 \\
0
\end{array}\right) .
\end{array}
$$

This set of equations has a nontrivial solution (i.e., $q_{x} \neq 0$, with $x=S, I$ ) only if the determinant of the matrix on the LHS is zero. To calculate the determinant, the complex field amplitudes $q_{x}$ are separated into real, positive amplitudes $b_{x}$ and real phases $\phi_{x}$ by setting $q_{x}=b_{x} e^{i \phi_{x}}$, and the complex parameter $\varepsilon$ is separated into a real, positive amplitude $\widetilde{\varepsilon}$ and a real phase $\theta$ by $\varepsilon=\widetilde{\varepsilon} e^{i \theta}$. For $\Delta$ this leads to the following condition for self-phase-locked operation:

$$
D^{2} b_{P}^{2}=\frac{1}{8}\left(2 \kappa_{S} \kappa_{I}-2 \Delta^{2}+5 \widetilde{\varepsilon}^{2}\right) \pm \sqrt{\alpha(\Delta)},
$$

where the abbreviation $\alpha$ is defined as 
TABLE I. Experimental parameter values for the two OPO setups used.

\begin{tabular}{ccccccc}
\hline \hline $\begin{array}{c}\text { Crystal length } \\
(\mathrm{mm})\end{array}$ & $\begin{array}{c}\kappa_{P} \\
(\mathrm{MHz})\end{array}$ & $\begin{array}{c}\kappa_{S} \\
(\mathrm{MHz})\end{array}$ & $\begin{array}{c}\kappa_{I} \\
(\mathrm{MHz})\end{array}$ & $\begin{array}{c}D \\
\left(10^{6} \mathrm{rad} / \mathrm{s}\right)\end{array}$ & $\begin{array}{c}G \\
\left(10^{6} \mathrm{rad} / \mathrm{s}\right)\end{array}$ & $\begin{array}{c}2 \delta \omega_{\text {lock }} \\
\left(10^{6} \mathrm{rad} / \mathrm{s}\right)\end{array}$ \\
\hline 38 & 258 & 249 & 13 & 1435 & 50 & 8.2 \\
60 & 243 & 236 & 11 & 630 & 14 & 3.1 \\
\hline \hline
\end{tabular}

$$
\alpha(\Delta):=-\frac{1}{16} \Delta^{2}\left[\left(\kappa_{S}+\kappa_{I}\right)^{2}+9 \widetilde{\varepsilon}^{2}\right]+\frac{1}{16} \widetilde{\varepsilon}^{2}\left(\kappa_{S} \kappa_{I}+\frac{9}{4} \widetilde{\varepsilon}^{2}\right) .
$$

Nontrivial solutions only exist if $\Delta$ is such that $\alpha(\Delta) \geqslant 0$, and the locking range corresponds to the range of the detuning $\Delta$ for which this condition is satisfied. As $\alpha(\Delta)$ is quadratic in $\Delta$ and has no linear components, and since the second derivative of $\alpha$ with respect to $\Delta$ is negative, the function $\alpha(\Delta)$ possesses a maximum at $\Delta=0$ and decreases symmetrically with the absolute value of $\Delta$. Therefore, the full locking range $2 \delta \omega_{\text {lock }}=6 \Delta_{\text {lock }}$ can be determined from Eq. (8) by setting $\alpha\left(\Delta_{\text {lock }}\right)=0$, resulting in

$$
\Delta_{\text {lock }}=\left(\frac{\widetilde{\varepsilon}^{2}\left(\kappa_{S}+\kappa_{I}+\frac{9}{4} \widetilde{\varepsilon}^{2}\right)}{\left(\kappa_{S}+\kappa_{I}\right)^{2}+9 \widetilde{\varepsilon}^{2}}\right)^{1 / 2} .
$$

In the experiment, according to Eq. (3), locking should thus occur if the two light frequencies $\omega_{S}$ and $\omega_{S^{\prime}}$ are (via a variation of $\Delta$ ) brought closer to each other than $\left|\omega_{S}-\omega_{S^{\prime}}\right|$ $\leqslant \delta \omega_{\text {lock }}=3 \Delta_{\text {lock }}$. Equation (9) shows that the locking range is a function of the OPO's construction and operating parameters $\kappa_{S}$ and $\kappa_{I}$, and of $\widetilde{\varepsilon}^{2}$. The latter parameter is given by $\widetilde{\varepsilon}^{2}=G^{2} b_{I}^{2}$, where $b_{I}^{2}=N_{I}$ is the intracavity idler photon number. Since in our experiment we can assume that $N_{I}$ is not noticeably depleted by idler SHG, the number of idler photons can be determined from the steady-state solutions of Eqs. (1) with $G$ set to zero (i.e., no idler SHG), which yields

$$
\widetilde{\varepsilon}^{2}=\frac{G^{2}}{D^{2}} \frac{\kappa_{S} \kappa_{P}}{4}\left(\frac{4|F| D}{\kappa_{P} \sqrt{\kappa_{S} \kappa_{I}}}-1\right)
$$

Using Eqs. (9) and (10), the locking range of the OPO divider can be predicted, if the cavity and crystal parameters $F$, $\kappa_{P}, \kappa_{S}, \kappa_{I}, D$, and $G$ are known. The pump rate $F$ can be determined from the incident pump power $P_{P}$ [see above Eq. (4)]. The remaining parameters $\kappa_{P}, \kappa_{S}, \kappa_{I}, D$, and $G$ usually differ from the values calculated from Eqs. (2), (4), and (5), mainly due to the mean-field and plane-wave approximation and an eventual phase mismatch. However, a convenient way to determine these parameters is to extract them from independent measurements. First we derived the cavity parameters $\kappa_{P}, \kappa_{S}$, and $\kappa_{I}$ by fitting the output characteristics of a triply resonant $\mathrm{cw}$ OPO to the experimental measurements, using slope and pump power at threshold as fitting parameters, as is described in Ref. [16]. In our experiment, the output power was measured using output coupling mirrors with different, well-known reflectivities, while the idler SHG was tuned off phase matching via the crystal temperature, so that the nonlinear coupling coefficient for the SHG process, $G$ in Eqs. (1), could be set to zero. The nonlinear coupling coefficient for the OPO process $D$ was then determined from the measured pump power at threshold. Finally, after having tuned back the crystal temperature to phase matching of the idler SHG, the power ratio of the frequency-doubled idler wave to the signal wave was measured, which was used to determine the nonlinear coupling coefficient $G$ valid in the experiments. The parameter values determined in the manner described are summarized in Table I. In our experiments, two different setups of the OPO divider were used, one with a 38-mm-long crystal and the other with a 60-mm-long crystal. Correspondingly, two sets of OPO parameters were derived as described and are given in Table I.

With these sets of experimental parameters, the theoretical values of the full locking range calculated by Eqs. (9) and (10) are $2 \delta \omega_{\text {lock }}=6 \Delta_{\text {lock }}=8.2 \times 10^{6}$ and $3.1 \times 10^{6} \mathrm{rad} / \mathrm{s}$, corresponding to a frequency-locking range of 1.3 and $0.5 \mathrm{MHz}$ for the divider with the 38 and the 60-mm-long crystal, respectively.

To this end, it is worth noticing that in steady state, from Eq. (7) one finds two different solutions for the intracavity pump field. As above threshold the intracavity pump photon number $N_{P}$ of a cw OPO is clamped to its threshold value $N_{\text {th }}$ [16], these two solutions belong to two different pump powers at threshold, which can be calculated from Eq. (7) with $N_{\mathrm{th}}=N_{P}=b_{P}^{2}$ :

$$
N_{\mathrm{th}}^{(1,2)}=\frac{1}{D^{2}}\left[\frac{1}{8}\left(2 \kappa_{S} \kappa_{I}-2 \Delta^{2}+5 \widetilde{\varepsilon}^{2}\right) \pm \sqrt{\alpha(\Delta)}\right] .
$$

According to this equation, the separation of the two values for the OPO threshold varies with the cavity detuning $\Delta$ (within the locking range). With the definition of $\alpha$ in Eq. (8), it can be shown that the difference of the threshold values is maximum at $\Delta=0$ and approaches zero at the edges of the locking range, i.e., where $\Delta=\Delta_{\text {lock }}$ with $\Delta_{\text {lock }}$ given by Eqs. (9) and (10). Furthermore, the difference between the high- and the low-threshold values increases with increasing $\widetilde{\varepsilon}^{2}$, which is proportional to $G^{2}$, as given in Eq. (10). Therefore, a stronger optical coupling between the signal and idler fields (via the idler SHG process) results in an increased difference in the threshold photon number.

A similar threshold splitting of a cw OPO has been qualitatively observed in an experiment by Mason et al. [9] and has been described theoretically by Fabre et al. [15]. In Mason's experiment, a type-II OPO (perpendicularly polarized signal and idler) was operated at frequency degeneracy as a frequency by 2 divider, by injecting a small fraction of the 
signal into the idler field and vice versa. However, so far we have not observed such a threshold splitting in our experiment with the by 3 divider.

\section{Frequency stability}

By solving the coupled field equations in the steady-state regime, we derived expressions for the locking range and the oscillation threshold. These results give the conditions that need to be satisfied in order to obtain phase-locked frequency division by 3 . In the following, we investigate the steadystate solutions of the phases of the signal and idler output fields with respect to the input pump phase and the detuning. Thereafter we derive the stability of frequency division, i.e., an expression for the residual time-averaged phase fluctuations of the self-phase-locked OPO.

By separating the complex values into positive real amplitudes and real phases in Eqs. (6), the steady-state phases for $q_{S}$ and $q_{I}$ can be rewritten as

$$
\begin{gathered}
\sin \left(\phi_{S}+\phi_{I}-\phi_{P}\right)=\frac{1}{3 D b_{P}}\left(\kappa_{S} r+\frac{\kappa_{I}}{2 r}\right), \\
\cos \left(\phi_{S}+\phi_{I}-\phi_{P}\right)=\frac{1}{D b_{P}}\left(\Delta r+\frac{\Delta}{2 r}\right), \\
\sin \left(\phi_{S}-\phi_{I}-\theta\right)=\frac{1}{\widetilde{\varepsilon}}\left(\Delta r+\frac{\Delta}{r}\right), \\
\cos \left(\phi_{S}-\phi_{I}-\theta\right)=\frac{1}{3 \widetilde{\varepsilon}}\left(\kappa_{S} r-\frac{\kappa_{I}}{r}\right),
\end{gathered}
$$

where we have used the abbreviation $r:=b_{S} / b_{I}$. Note that for the case of a cw OPO without idler SHG, i.e., for $\widetilde{\varepsilon}=0$ and $\Delta=0$, it can be easily verified from Eqs. (12) that the signal-idler sum phase is given by $\phi_{S}+\phi_{I}=\phi_{P}+\pi / 2$, while the difference phase $\phi_{S}-\phi_{I}$ can assume an arbitrary value, as known from standard OPO theory $[3,17]$.

For a determination of $r$ in Eq. (12), we use Eq. (7) and $\cos ^{2} x+\sin ^{2} x=1$ to obtain

$$
\left(\kappa_{S}^{2}+9 \Delta^{2}\right) r^{4}-( \pm 24 \sqrt{\alpha}) r^{2}-\left(\kappa_{I}^{2}+9 \Delta^{2}\right)=0 .
$$

In this equation, the positive sign of the term with $\sqrt{\alpha}$ corresponds to the high-threshold state of the OPO, and the negative sign to the low-threshold state. For the lowthreshold state, one obtains

$$
r^{2}=\frac{-24 \sqrt{\alpha} \pm \sqrt{\beta}}{2\left(\kappa_{S}^{2}+9 \Delta^{2}\right)},
$$

where the abbreviation $\beta$ is defined as $\beta:=(24 \sqrt{\alpha})^{2}+4\left(\kappa_{S}^{2}\right.$ $\left.+9 \Delta^{2}\right)\left(\kappa_{I}^{2}+9 \Delta^{2}\right)$. Note that the condition $r^{2} \geqslant 0$ already determines the sign of $\sqrt{\beta}$, depending on the choice of $\alpha$ and $\beta$ (via a choice of $\Delta$ ).

By dividing the respective sine and cosine equations (12) by each other, the signal-idler sum and difference phases can be calculated as

$$
\begin{gathered}
\phi_{S}+\phi_{I}=\phi_{P}+\operatorname{arccot}\left[\frac{3 \Delta(r+1 / 2 r)}{\kappa_{S} r+\kappa_{I} / 2 r}\right]+2 m \pi, \\
\phi_{S}-\phi_{I}=\theta+\arctan \left[\frac{3 \Delta(r+1 / r)}{\kappa_{S} r-\kappa_{I} / r}\right]+2 n \pi .
\end{gathered}
$$

The integer numbers $m$ and $n$ in these equations take into account that the phases are defined only modulo $2 \pi$.

Equations (15) show that the sum phase of the signal and idler fields follows the input pump phase. This behavior is well known for a standard OPO. In our divider, however, the difference phase is also locked, i.e., it follows the phase $\theta$ of the coupling parameter $\varepsilon$. Furthermore, from the definition of $\varepsilon:=i G q_{I}$, with the approximation of weak idler SHG, it follows that $\theta=\phi_{I}+\pi / 2$; thus the idler phase can be written as

$$
\phi_{I}=\frac{1}{3} \phi_{P}+l \frac{2 \pi}{3}+f_{I}(\Delta) .
$$

In this expression, $f_{I}(\Delta)$ is defined as

$$
\begin{aligned}
f_{I}(\Delta):= & \frac{1}{3} \operatorname{arccot}\left[\frac{3 \Delta(r+1 / 2 r)}{\kappa_{S} r+\kappa_{I} / 2 r}\right]-\frac{1}{3} \arctan \left[\frac{3 \Delta(r+1 / r)}{\kappa_{S} r-\kappa_{I} / r}\right] \\
& -\frac{\pi}{6}
\end{aligned}
$$

and $l$ is an integer number. Similarly, the phase of the signal wave can be written as

$$
\phi_{S}=\frac{2}{3} \phi_{P}+k \frac{2 \pi}{3}+f_{S}(\Delta)
$$

where $f_{S}(\Delta)$ is defined as

$$
\begin{aligned}
f_{S}(\Delta):= & \frac{2}{3} \operatorname{arccot}\left[\frac{3 \Delta(r+1 / 2 r)}{\kappa_{S} r+\kappa_{I} / 2 r}\right]+\frac{1}{3} \arctan \left[\frac{3 \Delta(r+1 / r)}{\kappa_{S} r-\kappa_{I} / r}\right] \\
& +\frac{\pi}{6},
\end{aligned}
$$

with an integer number $k$.

The physical meaning of Eqs. (16) and (18) can be described as follows. The OPO internal pump field is defined by the external pump laser. The signal and idler phases can assume one of three distinct phase eigenvalues, which are separated by $2 \pi / 3$ and numbered by $l$. We note that this agrees with an earlier prediction where an all-optical divider by $n$ should possess $n$ phase eigenvalues [17]. Our numerical calculations have shown that the initial conditions during startup of the OPO, such as imposed through vacuum fluctuations, determine which of the three phase eigenvalues will be assumed in steady state.

The precision of the frequency division, however, is determined by a residual frequency instability, which could be caused as follows. In an experiment, the frequency $\omega_{P}$ and thus its exact one-third $\omega_{P} / 3=\Omega_{I}$, is set by the pump laser. Nevertheless, as the pump laser frequency is subject to a residual thermal drift, the cavity detuning $\Delta$ for the signal 
and idler waves might drift as well, because the length of the OPO cavity is locked to the pump laser wavelength (via a Pound-Drever-Hall servo loop; see Sec. III). Furthermore, this electronic servo loop possesses only finite response time, which is in our experiments approximately $1 \mathrm{~ms}$ given by the piezoelectric transducer. Acoustic perturbations with frequencies above $1 / \mathrm{ms}$ may thus rapidly change the cavity length, leading to a small and random fluctuation of $\Delta$ as a function of time.

As $\Delta$ enters the eigenvalues assumed by the divider in steady state [via $f_{I}$ and $f_{S}$ in Eqs. (16) and (18)], these eigenvalues are expected to drift and fluctuate as well. A temporal change of an eigenvalue $\phi_{x}(t)$, however, corresponds, via $d \phi_{x}(t) / d t$, to a frequency deviation of the signal and idler frequencies from the exactly divided values.

In our experiments, we indeed observed such a slowly drifting frequency deviation (when the OPO was not selfphase-locked), whereas faster fluctuations could not be measured. Therefore we assume that the observed drift is dominating any residual rapid frequency fluctuations, and in the following considerations we concentrate on a slow drift of the cavity detuning $\Delta$ as the origin of a residual frequency instability of the divider.

A measure for a residual frequency instability of the frequency divider is the full width at half maximum $\left(\mathcal{W}_{\text {FWHM }}\right)$ of the power spectrum of the beat between the generated idler frequency and the exact one-third of the pump frequency $\mathcal{W}_{\mathrm{FWHM}}\left(\omega_{I}-\Omega_{I}\right)$. First, we derive an expression for this deviation of the idler frequency of the exactly divided pump frequency by assuming that the cavity detuning $\Delta$ varies as a function of time as described. By assuming that $d \Delta / d t \neq 0$ and taking the time derivative of Eq. (16), we find a beat frequency changing with time:

$$
\omega_{I}-\Omega_{I}=\omega_{I}-\frac{1}{3} \omega_{P}=\frac{d}{d t}\left(\phi_{I}-\frac{1}{3} \phi_{P}\right)=\frac{d}{d t} f_{I}(\Delta),
$$

where

$$
\frac{d}{d t} f_{I}(\Delta)=\frac{d f_{I}}{d \Delta} \frac{d \Delta}{d t}
$$

The $\mathcal{W}_{\text {FWHM }}$ spectral width of this beat is given by the mean square value of the difference phase $\psi_{I}:=\phi_{I}(t)-\frac{1}{3} \phi_{P}(t)$ divided by the measurement time period $T$ [3]:

$$
\mathcal{W}_{\mathrm{FWHM}}\left(\omega_{I}-\Omega_{I}\right)=\left\langle\left[\psi_{I}(T)-\psi_{I}(0)\right]^{2}\right\rangle \frac{1}{T},
$$

where the angular brackets denote the statistical mean value over many periods $T$. Instead of $\left\langle\left[\psi_{I}(T)-\psi_{I}(0)\right]^{2}\right\rangle$ we can write $\left\langle\left[f_{I}(\Delta(T))-f_{I}(\Delta(0))\right]^{2}\right\rangle$ [see Eq. (20)], where for $f_{I}(\Delta)$ we insert the RHS of Eq. (21), after carrying through an integration over time. The first factor on the RHS of Eq. (21) is constant with respect to time and can be extracted from the mean square values, and we obtain

$$
\mathcal{W}_{\mathrm{FWHM}}\left(\omega_{I}-\Omega_{I}\right)=\left(\frac{d f_{I}}{d \Delta}\right)^{2} \frac{\left\langle[\Delta(T)-\Delta(0)]^{2}\right\rangle}{T} .
$$

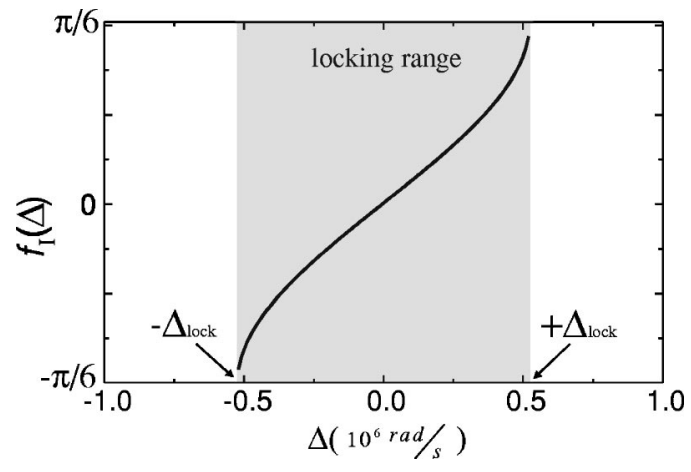

FIG. 3. Idler phase shift $f_{I}$ versus the detuning $\Delta$, as given by Eq. (17), and calculated for the experimental parameters given in Table I for the OPO with the 60-mm-long crystal.

In the following, we use Eq. (23) to predict an absolute quantitative value for the width of the beat between the generated idler wave and the desired wave with exactly one-third of the pump frequency, as would be expected for our experimental setup. For this prediction, we use the parameters of the setup with the 60-mm-long crystal (given in Table I), because frequency division with this setup was measured to be more stable than with the setup with the 38-mm crystal.

To calculate a quantitative value for the width of the beat, we need to retrieve two numbers for the two factors on the RHS of Eq. (23). The first factor $\left(d f_{I} / d \Delta\right)^{2}$ can be derived analytically from Eq. (17). Therefore, we calculated the function $f_{I}(\Delta)$ given by Eq. (17), as plotted as a function of the detuning in Fig. 3. It can be seen that the value of $f_{I}(\Delta)$ varies over $\pi / 3$ within the theoretically expected locking range, i.e., for $\Delta \leqslant\left|\Delta_{\text {lock }}\right|=0.52 \times 10^{6} \mathrm{rad} / \mathrm{s}$, which is indicated as gray background. Note that in the center of the locking range the function $f_{I}(\Delta)$ shows a linear dependence on $\Delta$ with a slope of $d f_{I} / d \Delta=6.5 \times 10^{-7} \mathrm{~s}$, while at the edges of the locking range a nonlinear dependence is observed. Using this slope around $\Delta=0$, we obtain the value $\left(d f_{I} / d \Delta\right)^{2}=4.2 \times 10^{-13} \mathrm{~s}^{2}$ for the first factor.

The second factor $\left\langle[\Delta(T)-\Delta(0)]^{2}\right\rangle(1 / T)$ is retrieved from an experiment where the drift of the beat frequency between the signal wave and the frequency-doubled idler wave was measured, i.e., the drift of the difference $\left(\omega_{S}\right.$ $\left.-2 \omega_{I}\right)=3 \Delta$, when the OPO was not self-phase-locked. This experiment will be presented in more detail in the next section. In a radio frequency (rf) measurement of this beat, a drift of the beat frequency of typically $2 \mathrm{MHz}$ in a time period of $T=60 \mathrm{~s}$ was observed. This corresponds to a value of $[\Delta(T)-\Delta(0)]^{2}(1 / T)=2.9 \times 10^{11} \mathrm{rad}^{2} / \mathrm{s}^{3}$ for the second factor of Eq. (23).

Using these values for the two factors on the RHS of Eq. (23), the spectral linewidth of the phase-locked OPO is calculated to be $\mathcal{W}_{\mathrm{FWHM}}\left(\omega_{I}-\Omega_{I}\right)=0.12 \mathrm{rad} / \mathrm{s}$. At the angular frequency of the idler wave of $\Omega_{I}=774 \times 10^{12} \mathrm{rad} / \mathrm{s}$ (wavelength of $2436 \mathrm{~nm}$ ), this corresponds to a relative frequency instability of $\mathcal{W}_{\text {FWHM }}\left(\omega_{I}-\Omega_{I}\right) / \Omega_{I}=1.6 \times 10^{-15}$ in $60 \mathrm{~s}$.

A direct measurement of this residual frequency instability would require first frequency-tripling the OPO's idler output wave and then superimposing it on the OPO's input pump frequency, to obtain a beat frequency in the rf range. In 
our experiment, however, we found it technically easier to perform a beat measurement in a different manner, i.e., by superimposing the second harmonic of the OPO's signal wave output on the sum frequency of the pump input and the OPO idler output wave.

This measurement of $\mathcal{W}_{\text {FWHM }}\left[2 \omega_{S}-\left(\Omega_{P}+\omega_{I}\right)\right]$ (see Sec. III) compares the frequency of the OPO output waves $2 \omega_{S}$ $-\omega_{I}$ with that of the OPO input wave $\Omega_{P}$. Because the phases of both the signal and the idler waves vary linearly with $\Delta$ in the center of the locking range, the frequency instability of this comparison is proportional to the frequency instability of the idler output wave with respect to the exact one-third of the pump wave. To derive the corresponding proportionality factor, we use Eqs. (16) and (18) to express the time dependence of the beat frequency $2 \omega_{S}-\left(\Omega_{P}\right.$ $\left.+\omega_{I}\right)$, i.e.,

$$
\left(2 \omega_{S}-\omega_{I}\right)-\Omega_{P}=\frac{d}{d t}\left[2 f_{S}(\Delta)-f_{I}(\Delta)\right] .
$$

After carrying out the same steps as above we obtain the spectral bandwidth of this beat as

$$
\begin{aligned}
& \mathcal{W}_{\text {FWHM }}\left[\left(2 \omega_{S}-\omega_{I}\right)-\Omega_{P}\right] \\
& =\left(2 \frac{d f_{S}}{d \Delta}-\frac{d f_{I}}{d \Delta}\right)^{2} \frac{[\Delta(T)-\Delta(0)]^{2}}{T} .
\end{aligned}
$$

In the center of the locking range, the derivatives $d f_{S} / d \Delta$ and $d f_{I} / d \Delta$ are constant with values of $d f_{S} / d \Delta=-7.4$ $\times 10^{-7} \mathrm{~s}$ and $d f_{I} / d \Delta=6.5 \times 10^{-7} \mathrm{~s}$. With $[\Delta(T)$ $-\Delta(0)]^{2}(1 / T)$ as derived above we calculate $\mathcal{W}_{\text {FWHM }}\left[\left(2 \omega_{S}-\omega_{I}\right)-\Omega_{P}\right]=1.3 \mathrm{rad} / \mathrm{s}$. Dividing the value for $\mathcal{W}_{\text {FWHM }}\left[\left(2 \omega_{S}-\omega_{I}\right)-\Omega_{P}\right]$ by the value for $\mathcal{W}_{\text {FWHM }}\left(\omega_{I}\right.$ $-\Omega_{I}$ ) gives the desired proportionality factor:

$$
\frac{\mathcal{W}_{\mathrm{FWHM}}\left[\left(2 \omega_{S}-\omega_{I}\right)-\Omega_{P}\right]}{\mathcal{W}_{\mathrm{FWHM}}\left(\omega_{I}-\Omega_{I}\right)}=\frac{1.3}{0.12}=10.8
$$

As a result of these calculations we expect for the OPO setup with the 60-mm-long crystal a spectral bandwidth of the beat frequency $\left(2 \omega_{S}-\omega_{I}\right)-\Omega_{P}$ of $1.3 \mathrm{rad} / \mathrm{s}$, which corresponds to a spectral bandwidth of a direct idler beat measurement $\omega_{I}-\Omega_{I}$ of $0.12 \mathrm{rad} / \mathrm{s}$. The latter value means that the OPO should be able to divide the pump laser frequency by 3 with a fractional frequency instability of $1.6 \times 10^{-15}$.

\section{EXPERIMENTAL SETUP AND RESULTS}

The experimental setup of the OPO frequency divider is similar to the one described in Refs. [10] and [11]. The frequency to be divided is provided by a single-frequency AlGaAs diode master-oscillator power-amplifier (MOPA) system [18]. It provides a near diffraction-limited output beam with a maximum power of $340 \mathrm{~mW}$ at a wavelength of 812 $\mathrm{nm}$ for pumping the OPO. The diode MOPA wavelength can be grating tuned over several nanometers and continuously fine-tuned (without mode hops) over $1 \mathrm{GHz}$ via a piezo control of the diode oscillator cavity length.

For division of the diode laser frequency by 3 , we use a triply resonant two-mirror $\mathrm{OPO}$, which is based on quasiphase-matching (QPM) in a periodically poled $\mathrm{LiNbO}_{3}$ (PPLN) crystal. Two different crystals were available for the experiment, having lengths of 38 and $60 \mathrm{~mm}$, respectively. Each crystal carries two sections with different poling periods as shown in Fig. 1. The first section with length $L_{1}$ carries a poling period of $\Lambda_{1}$ to quasi-phase-match parametric down-conversion of 812 -nm radiation to a signal wavelength of $1218 \mathrm{~nm}$ and an idler wavelength of $2436 \mathrm{~nm}$, and the second section with length $L_{2}$ carries a poling period of $\Lambda_{2}$ for quasi-phase-matching the frequency doubling of the idler wave. In the first crystal, $L_{1}=29 \mathrm{~mm}$ and $L_{2}=9 \mathrm{~mm}$, and in the second crystal $L_{1}=40 \mathrm{~mm}$ and $L_{2}=20 \mathrm{~mm}$. In both crystals, the poling periods are $\Lambda_{1}=21.2 \mu \mathrm{m}$ and $\Lambda_{2}$ $=34.1 \mu \mathrm{m}$, so that the OPO and the SHG process are simultaneously phase matched at a crystal temperature of about $167^{\circ} \mathrm{C}$. Both PPLN crystals are $0.5 \mathrm{~mm}$ thick, and their faces are antireflection coated for the pump, signal, and idler wavelengths around 812,1218, and $2436 \mathrm{~nm}$, respectively (residual reflectivity specified to be less than $0.1 \%$ ).

First, the 38-mm-long PPLN crystal is placed at the center of a symmetric two-mirror linear cavity, where both mirros have a radius of curvature of $50 \mathrm{~mm}$, and are separated by $115 \mathrm{~mm}$. The input mirror M1 has a pump transmission of $4 \%$ and is highly reflective for the signal and idler wavelengths $(R>99.7 \%)$. The output mirror M2 has a signal transmission of $20 \%$ and is highly reflective for the pump and idler wavelengths $(R>98.5 \%)$. For the second experiment with the 60-mm-long PPLN crystal, we used the same OPO cavity except that a higher pump wave transmission at M1 of $8 \%$ is chosen and the separation between the mirrors is increased to $125 \mathrm{~mm}$. In both setups, the pump beam is mode matched to the OPO cavity mode with a spherical lens of $75 \mathrm{~mm}$ focal length, resulting in a beam radius of approximately $30 \mu \mathrm{m}$ at the focus. To provide stable cw operation, the cavity length is stabilized to the pump wavelength with a piezo transducer (PZT) attached to mirror M2 by using the Pound-Drever-Hall technique $[10,19]$.

Figure 4 shows the output power of both OPOs at the signal wavelength as a function of pump power (38-mm crystal, circles; 60-mm crystal, triangles), as measured behind the output mirror M2 at a crystal temperature of around $167^{\circ} \mathrm{C}$. For the 38 -mm-long crystal setup, the pump power at threshold is approximately $40 \mathrm{~mW}$ and the maximum signal output power is $23 \mathrm{~mW}$ at a pump power of $320 \mathrm{~mW}$. The residually transmitted idler output power behind M2 is measured to be less than $1 \mathrm{~mW}$ for this setup. For the $60-\mathrm{mm}$ crystal setup, the pump power at threshold is measured to be approximately $100 \mathrm{~mW}$, and the maximum signal output power is $38 \mathrm{~mW}$ with a maximum idler output power of 1.8 $\mathrm{mW}$.

The solid lines in Fig. 4 represent a best fit of the theoretically expected output power $P_{S}=\eta\left(\sqrt{P_{\mathrm{th}} P_{P}}-P_{\mathrm{th}}\right)$ to the experimental data as described in Ref. [16], where $\eta$ is the slope efficiency and $P_{\text {th }}$ is the threshold pump power. The best fit is achieved for $\eta=0.33$ and $P_{\mathrm{th}}=43 \mathrm{~mW}$ in the 38 mm-long crystal setup, and $\eta=0.50$ and $P_{\text {th }}=96 \mathrm{~mW}$ in the 60-mm-long crystal setup. These values are used to determine the experimental values of the parameters $\kappa_{P}, \kappa_{S}, \kappa_{l}$, 


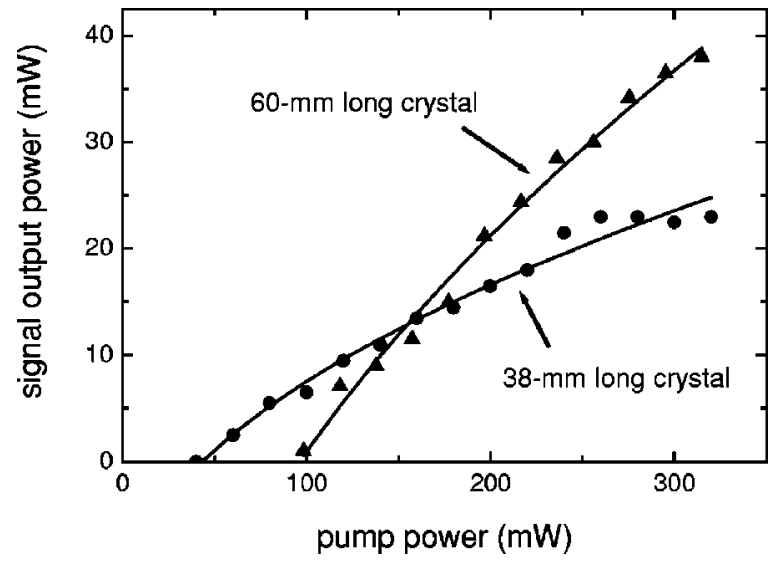

FIG. 4. Signal wave output power of the OPO as a function of pump power in the setup with the $38-\mathrm{mm}$ PPLN crystal (circles) and with the 60-mm PPLN crystal (triangles), together with the theoretical fit functions (solid lines). The pump, signal, and idler wavelengths are 812, 1218, and $2436 \mathrm{~nm}$, respectively, at a PPLN crystal temperature of about $167^{\circ} \mathrm{C}$.

and $D$, as listed in Table I, from which the theoretical locking range and phase stability of self-phase-locking are calculated, as was described in the previous section.

Using a double-grating optical spectrum analyzer (Ando AQ-6315A, spectral resolution $0.05 \mathrm{~nm}$ ), the emission of the signal wave and the frequency-doubled idler wave is observed simultaneously near a wavelength of $1218 \mathrm{~nm}$. The power ratio of the idler SHG wave to the signal wave is measured to be $-25 \mathrm{~dB}$ for the 38-mm-long crystal setup and $-33 \mathrm{~dB}$ for the $60-\mathrm{mm}$-long crystal setup. These values were used to determine the experimental value of the parameter $G$, as given in Table I. By changing the crystal temperature, the OPO can be tuned so that the signal wavelength coincides with the wavelength of the idler SHG within the resolution limit of the optical spectrum analyzer.

A higher spectral resolution is provided by a radio frequency beat measurement of the signal wave and the idler SHG wave. For measuring the beat spectrum, an InGaAs Schottky-type photodiode (New Focus model 1437, 3 dB bandwidth $35 \mathrm{GHz}$ ) is used, in combination with a rf spectrum analyzer with a frequency range of $9 \mathrm{kHz}$ to $26.5 \mathrm{GHz}$ (Hewlett-Packard model HP E4407B). Limited by the measurement bandwidth, the spectral width of the beat is measured to be smaller than $30 \mathrm{kHz}$ over an integration time of $10 \mathrm{~ms}$. A slow drift of the beat frequency can be observed (typically $2 \mathrm{MHz}$ in a time interval of $1 \mathrm{~min}$ for the $60-\mathrm{mm}$ crystal setup), while the power of the beat signal remains constant within $10 \%$. The observed drift mainly originates from slow thermal effects in the crystal, which can lead to a change of the optical cavity length. The 38-mm crystal setup shows a stronger drift of the beat frequency of typically 10 $\mathrm{MHz}$ per minute. A possible explanation of the higher stability of the setup with the 60-mm-long crystal compared to that with the 38-mm-long crystal is a thermally induced stabilization effect, as was reported in Ref. [20].

\section{A. Locking-range measurement}

In order to measure the locking range, the beat frequency is tuned toward zero, where the beat signal is expected to

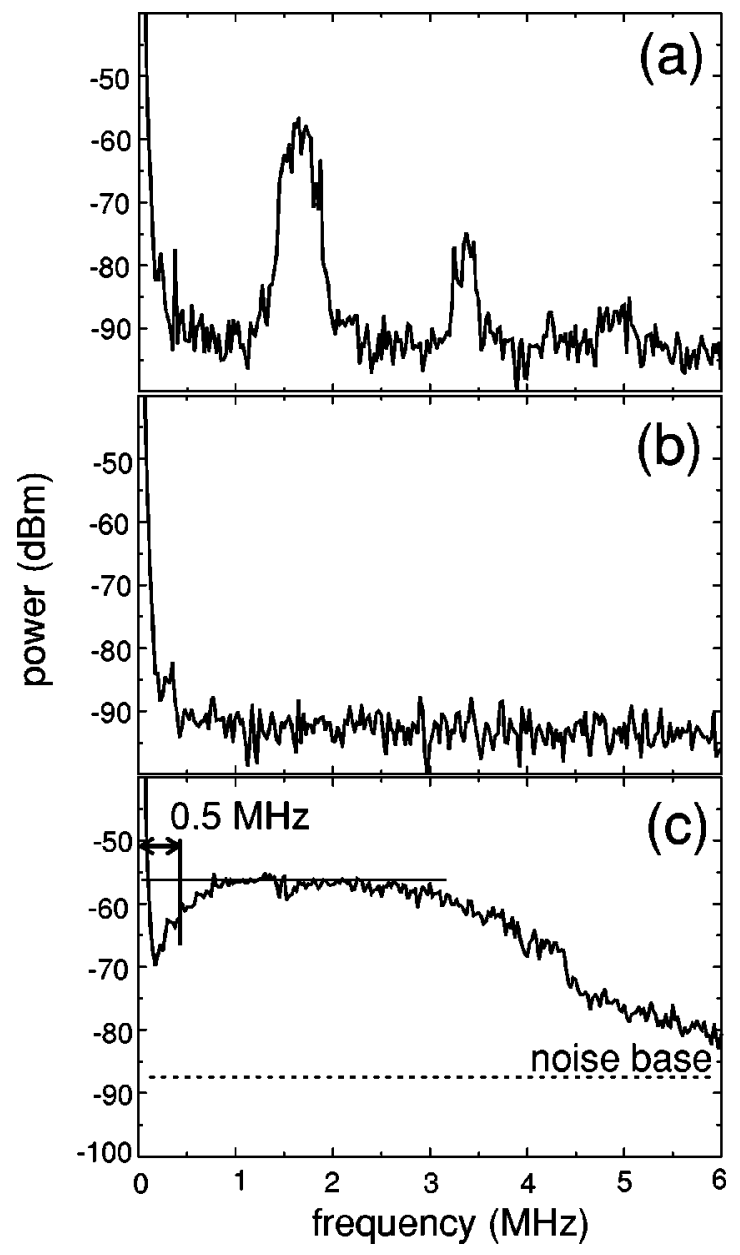

FIG. 5. rf beat spectra of the signal wave and the idler SHG waves for the OPO with the 38-mm crystal recorded near zero frequency with a rf spectrum analyzer (spectral resolution $30 \mathrm{kHz}$, sweep time $17.8 \mathrm{~ms}$ ). (a) is measured when the OPO is not phase locked, and (b) with the OPO being self-phase-locked. (c) is a maximum-hold trace for a hold time of $5 \mathrm{~min}$, showing a dip around zero frequency, the full width of which corresponds to a locking range of $1 \mathrm{MHz}$.

vanish when optical self-phase-locking occurs [10,11]. Tuning of the beat frequency is achieved by changing the crystal temperature by typically tenths of kelvin and by continuously tuning the pump frequency in the range of $100 \mathrm{MHz}$.

Figure 5 shows the rf beat spectra near zero frequency for the setup with the $38-\mathrm{mm}$-long crystal, measured while the OPO is not phase locked (a) in contrast to the situation when the OPO is self-phase-locked (b).

In Fig. 5(a), three peaks can be seen, a strong peak at about $1.5 \mathrm{MHz}$ and two weaker peaks at 3.5 and $5 \mathrm{MHz}$. There is also a peak at zero frequency, which is present in any rf spectrum analyzer measurement. The strong peak at $1.5 \mathrm{MHz}$ is the beat signal between the signal wave and the frequency-doubled idler wave, which indicates that the OPO is not phase locked. The additional peaks at higher frequencies can be explained as higher-harmonic distortion sidebands, which are expected from the laser injection locking theory for the case that the beat is outside but close to the locking range [7]. In qualitative agreement we observe these 
distortion sidebands only for smaller beat frequencies in the range of up to $10 \mathrm{MHz}$. All three peaks in Fig. 5(a) are broadened due to frequency fluctuations. When the beat is tuned toward zero values, as shown in Fig. 5(b), the beat signal (and its distortion sidebands) suddenly vanishes.

Figure 5(c) shows a maximum-hold trace recorded with a hold time of $5 \mathrm{~min}$, which allows determination of the locking range. The crystal temperature and thus the beat frequency drift slowly and randomly within the measured range. In the frequency range from 1 to $3 \mathrm{MHz}$ the strength of the beat signal does not change, while it decreases for frequencies higher than $3 \mathrm{MHz}$ as well as for frequencies lower than $1 \mathrm{MHz}$. The continuous tuning range of the beat is approximately $4.5 \mathrm{MHz}$. The beat signal decreases in the range of 3 to $4.5 \mathrm{MHz}$, and above $4.5 \mathrm{MHz}$ it jumps to a higher frequency, due to a mode hop of the signal and the idler frequency. In the range above $4.5 \mathrm{MHz}$ there is still a signal measurable above the noise level, which is caused by the distortion sidebands.

For the determination of the locking range, the frequency interval from near 0 to $1 \mathrm{MHz}$ is important. Here the maximum-hold trace shows a significant decrease of more than $10 \mathrm{~dB}$ in depth. This dip can be caused by suppression of the beat within the locking range due to injection locking, and it suggests a self-phase-locking of the OPO waves by intracavity idler SHG, as described also in Refs. [10] and [11]. The power values, which are below the top line by more than $6 \mathrm{~dB}$, give a half width of $0.5 \mathrm{MHz}$ in Fig. 5(c), which indicates a full width locking range of approximately $1 \mathrm{MHz}$. This agrees well with the theoretical value of 1.3 MHz calculated from Eqs. (7) and (8). In the same way, the locking range for the setup with the 60-mm-long crystal is measured to be $0.6 \mathrm{MHz}$, which also shows a good agreement with the theoretical value of $0.5 \mathrm{MHz}$.

\section{B. Phase-coherence measurement}

The observation of a suppressed beat within a certain frequency range in Fig. 5(c) is clear evidence of self-phaselocking and a useful method for measuring the locking range. However, since the beat signal vanishes in the phaselocked state, the phase stability of the frequency division cannot be obtained quantitatively from this measurement. For a quantitative measurement, the phases of the OPO output waves have to be compared with the phase of the pump input wave with an interferometric method. To make such an interferometric comparison possible, we convert, via external single-pass three-wave mixing, the input and output waves of the OPO into two light waves at the same wavelength. The setup of the input-output interferometer used and the wavelength conversion stages are schematically shown in Fig. 6.

The cw OPO frequency divider with the $38-\mathrm{mm}-$ long crystal shown in the upper part of Fig. 6 converts the pump wave at $812 \mathrm{~nm}$ from the diode MOPA pump laser to the signal wave at $1218 \mathrm{~nm}$ and the idler wave at $2436 \mathrm{~nm}$. The signal output wave is frequency doubled in single pass to generate a second-harmonic wave at $609 \mathrm{~nm}$. For this we use a 60-mm-long PPLN crystal with a poling period of $10.2 \mu \mathrm{m}$ at a crystal temperature of $42{ }^{\circ} \mathrm{C}$, which yields a maximum

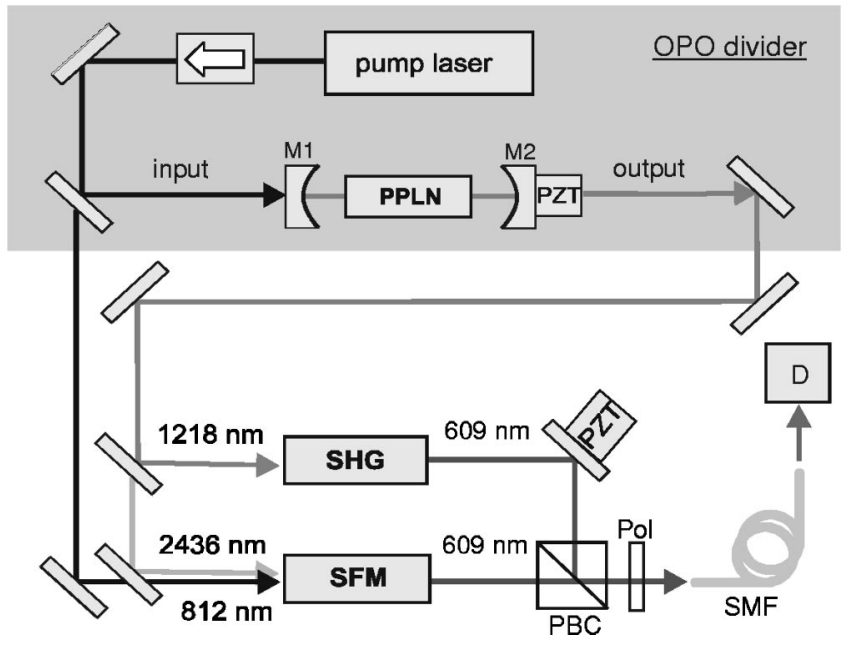

FIG. 6. Experimental setup for an interferometric measurement of the divider's phase stability. SHG, second-harmonic generation of the signal wave; SFM, sum-frequency mixing of the pump wave and the idler wave; PZT, piezo transducer; PBC, polarization beam combiner; Pol, polarizer; SFM, single-mode fiber; D, detector.

output power of $6 \mu \mathrm{W}$ at a signal wave input power of 35 $\mathrm{mW}$. A second wave at $609 \mathrm{~nm}$ is generated via sumfrequency mixing (SFM) using a part of the input pump wave and the idler output wave. For this we use a 40-mmlong PPLN crystal with a poling period of $12.67 \mu \mathrm{m}$ at a crystal temperature of $30.6{ }^{\circ} \mathrm{C}$. With $10 \mathrm{~mW}$ pump power at $812 \mathrm{~nm}$ and $1.5 \mathrm{~mW}$ idler power at $2436 \mathrm{~nm}$, a maximum power of approximately $100 \mathrm{nW}$ is generated. For each of the two conversion stages, a lens with a focal length of 125 $\mathrm{mm}$ is used to focus the OPO waves onto the PPLN crystals, and a lens with a focal length of $100 \mathrm{~mm}$ to collimate the 609 -nm waves behind the crystal. The two 609-nm waves are then compared interferometrically to obtain the phase coherence of the OPO output.

The frequency-doubled signal wave is first sent to a folding mirror, the position of which can be changed with a piezo transducer (PZT) to obtain an adjustable phase shift. The polarization of the frequency-doubled signal wave is rotated, such that its polarization is orthogonal to that of the pumpidler sum frequency. The waves are combined using a polarization beam combiner (PBC), and projected on a common linear polarization with a polarizer $(\mathrm{Pol})$. The linearly polarized superposition of the two beams is coupled into a $6.5-\mathrm{m}-$ long single-mode fiber (SMF), which serves as a spatial mode filter.

As the two 609-nm beams are derived from two different nonlinear processes, a temporarily stable interference pattern behind the fiber can only be expected if the frequency division is exact, i.e., phase coherent. Otherwise, the two 609-nm waves would beat with a frequency $\left|\left(2 \omega_{S}\right)-\left(\Omega_{P}+\omega_{I}\right)\right|$ $=|\delta \omega|$. In a previous experiment we indeed observed a stable interference pattern with a charge-coupled device camera [21], which qualitatively proved the phase coherence of the frequency divider. In the current experiment, however, a quantitative measurement of the divider's phase coherence was obtained as follows. 


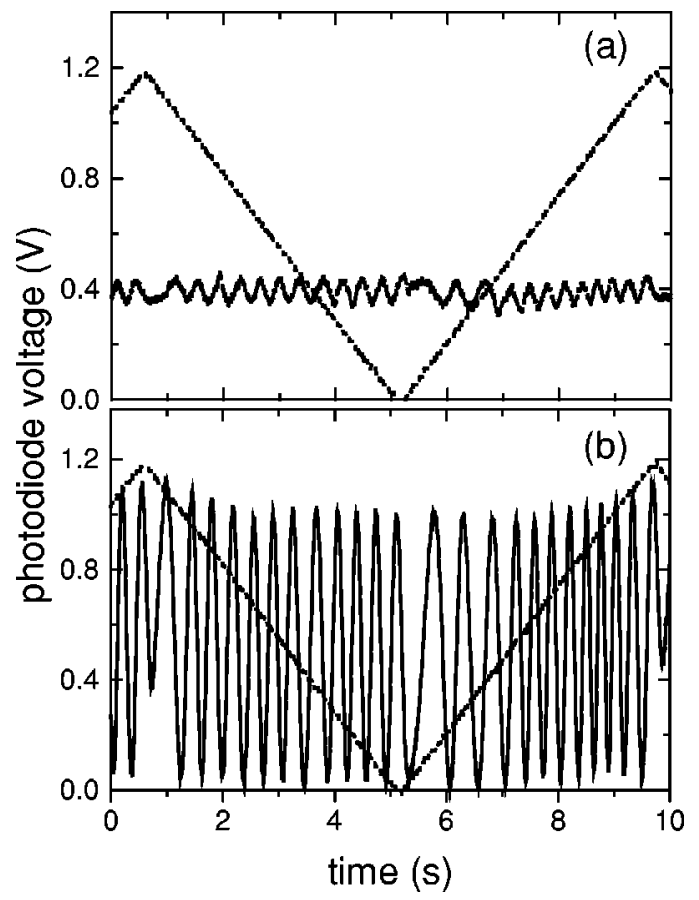

FIG. 7. Photodiode signal of the superimposed 609-nm beams generated by signal SHG and pump-idler SFM as a function of time, measured after transmission through a single-mode fiber. The dashed line gives the voltage ramp applied to the PZT for varying the interferometer path length. (a) is measured with the free-running OPO, and (b) is recorded while the OPO is self-phase-locked.

After passing the fiber, the two 609-nm beams have the same spatial mode, and thus can be focused onto a Si photodiode with well-matched phase fronts. If the frequency division by 3 is perfectly phase coherent, and if the intensities of both beams are perfectly balanced by a suitable rotation of the polarizer in front of the fiber, the contrast of the interference pattern can be expected to be $100 \%$. To measure the contrast, the path length of one interferometer arm is varied by applying a periodic voltage ramp to the PZT.

The measured photodiode voltage signal, which is proportional to the optical power, is plotted in Fig. 7 as a function of time. This measurement is performed with the OPO setup with the 38-mm-long crystal. Figure 7(a) shows a photodiode signal while the OPO is not phase locked, and it was phase locked while the signal shown in Fig. 7(b) was recorded. For reference, the voltage ramp applied to the PZT for varying the path length is shown as dashed lines. As can be seen, the induced change of the phase difference between the two $609-\mathrm{nm}$ waves results in a modulation of the interferometer output intensity, varying between the minimum value $I_{\text {min }}$ and the maximum value $I_{\max }$. The contrast of the interference is defined as $K:=\left(I_{\max }-I_{\min }\right) /\left(I_{\max }+I_{\min }\right)$, which is a direct measure of the coherence of the two 609-nm waves.

When the OPO is not phase locked, the interferometer output gives a constant signal with a small modulation with $K<15 \%$, as shown in Fig. 7(a). This residual modulation is caused by the interference between the pump-idler SFM wave and a third wave at $609 \mathrm{~nm}$ obtained from SFM of the signal and the idler SHG wave. The last process is phase matched in the signal SHG crystal, and the idler SHG wavelength cannot be filtered easily, because it is very close to the signal wavelength. This resulting third $609-\mathrm{nm}$ wave possesses the same frequency as the pump-idler SFM wave, and therefore shows interference independent from the phaselocking state of the frequency divider.

In contrast, when the OPO is phase locked, the interference pattern possesses a contrast of $K \approx 100 \%$, as shown in Fig. 7(b). This is evidence of perfect phase coherence of the two 609-nm waves, and, consequently, of phase-coherent division of the pump laser frequency by 3 with the self-phaselocked cw OPO. Smaller changes of the contrast in Fig. 7(b) are caused by power fluctuations of each of the 609-nm beams, which lead to a change of the intensity balance.

\section{Frequency-stability measurement}

In principle, a quantitative measurement of the frequency stability of the division by 3 should be possible with the described setup, by turning off the PZT voltage and measuring the photodiode signal as a function of time. However, we found that this does not allow a good discrimination between the frequency noise to be measured and intensity fluctuations of the two 609-nm waves. Therefore, we modified the setup shown in Fig. 6. An acousto-optic modulator (AOM, Isomet IMD-80) is used to shift the frequency of the frequencydoubled signal wave by $80 \mathrm{MHz}$. As before, it is superimposed on the pump-idler SFM wave. Behind the fiber, the light power is detected with a photodiode $(3 \mathrm{~dB}$ bandwidth $1.5 \mathrm{GHz})$. The photodiode signal is amplified with a lownoise rf amplifier (Miteq model AU-1310, $32.4 \mathrm{~dB}$ gain from $10 \mathrm{kHz}$ to $500 \mathrm{MHz}$ ) and recorded by the rf spectrum analyzer. With this modified setup, the near-zero frequency beat signal is shifted to the range of $80 \mathrm{MHz}$, where a much lower noise level enables beat frequency measurements with a considerably improved quality. If the OPO is self-phase-locked and the frequency division is phase coherent, a stable and narrow peak is expected at the AOM frequency.

Figure 8 shows examples of the measured rf spectra around the AOM frequency of $80 \mathrm{MHz}$ (solid lines), for a number of different spectral resolution bandwidths, and averaging over 20 measurements. The spectra are measured for the OPO with the 60-mm-long crystal, which was more stable than the setup with the 38 -mm-long crystal. The dashed lines in Fig. 8 give the electronic background signal obtained with the photodiode blocked (dark signal). Here the $80-\mathrm{MHz}$ peak, which is typically $10 \mathrm{~dB}$ below the optical signal, is caused by rf pickup from the oscillator used in the AOM. The low-frequency sidebands separated by $50 \mathrm{~Hz}$, which are observed at maximum resolution in the beat signal as well as in the dark signal, are caused by a pickup from the ac power line.

The narrow spectral bandwidth of the rf spectra in Fig. 8 shows that the divider operates with high precision in all cases. The linewidth of all photodiode signals is found to be equal to the resolution bandwidth of the spectrum analyzer, down to the highest resolution chosen. From Fig. 8(c) we obtain as an upper limit for the stability of the OPO divider a bandwidth of $10 \mathrm{~Hz}$ for an averaging time of $10 \mathrm{~s}$ (corre- 

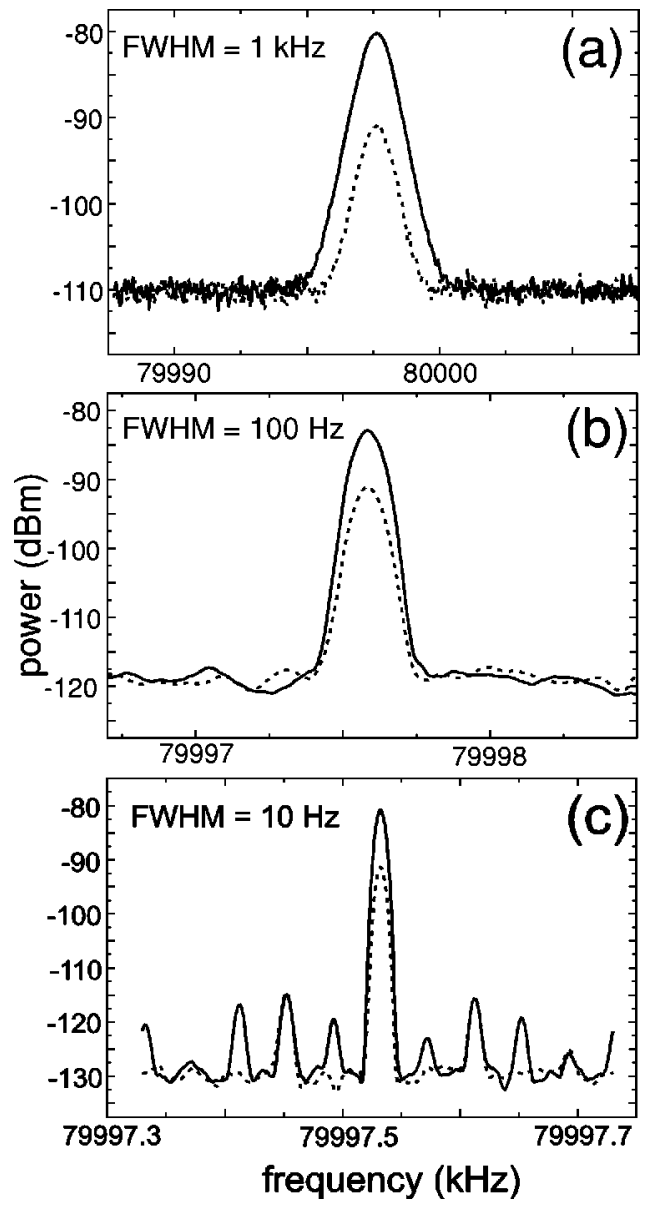

FIG. 8. rf beat spectra of two 609-nm waves generated by signal SHG and pump-idler SFM at the frequency of the AOM, recorded with a rf spectrum analyzer (averaging over 20 sweeps, spectral resolution (a) $1 \mathrm{kHz}$ with a sweep time of $275 \mathrm{~ms}$, (b) $100 \mathrm{~Hz}$ with $300 \mathrm{~ms}$, and (c) $10 \mathrm{~Hz}$ with $480 \mathrm{~ms}$ ). The dashed lines show the background spectra recorded with the photodiode blocked.

sponding to 20 measurements with a sweep time of $480 \mathrm{~ms}$ each). This value represents the frequency instability of the OPO output waves $2 \omega_{S}-\omega_{I}$ compared to the OPO input pump wave $\Omega_{P}$. Using Eq. (26), this corresponds to a residual instability of the idler wave of $5.8 \mathrm{rad} / \mathrm{s}$, or a fractional frequency instability of the by-3 divider of $7.6 \times 10^{-14}$ for a measurement time of $10 \mathrm{~s}$. As this value is limited by the resolution of the experimental recording technique, it is consistent with the theoretically expected value of $1.6 \times 10^{-15}$, as calculated with Eq. (25) in the previous section. The stability of the divider has not been systematically recorded on a longer time scale so far, but in our measurements we have observed up to $15 \mathrm{~min}$ of uninterrupted phase locking.

\section{SUMMARY}

Phase-coherent division of an optical frequency by 3 is realized with an all-optical frequency divider based on a self- phase-locked cw OPO. The performance of such self-phaselocking is investigated theoretically and experimentally.

For a theoretical description of the divider, we solve the coupled field equations of the OPO-SHG system in the steady-state regime. Analytical expressions for the locking range and the frequency stability of the self-phase-locked OPO are given and used to calculate the values expected for the current experiment. The theoretically calculated fractional frequency instability of the frequency division is as high as $1.6 \times 10^{-15}$ with an expected locking range of about $1 \mathrm{MHz}$.

In the experiment, the frequency to be divided is provided by a diode MOPA system operating at a wavelength of 812 $\mathrm{nm}$. The cw OPO is based on QPM in a PPLN crystal, in which two differently poled sections serve for simultaneous phase matching of two different nonlinear processes. The first section provides QPM for parametric oscillation at a signal wavelength of $1218 \mathrm{~nm}$ and an idler wavelength of $2436 \mathrm{~nm}$. The second section provides QPM for SHG of the idler wave. Optical injection locking of the OPO by intracavity idler SHG is proved by measuring the suppression of the beat note between the signal wave and the frequencydoubled idler wave. The locking range is determined to be up to $1 \mathrm{MHz}$. This agrees with the theoretical prediction.

In order to further investigate the self-phase-locking, the relative phases of the pump, signal, and idler waves are measured by using an interferometric setup with nonlinear wavelength conversion. Phase coherence among the three OPO waves is verified by measuring a stable interference pattern with a contrast of $100 \%$, which is observed over a time period of up to $15 \mathrm{~min}$. With a rf beat experiment where one of the two interfering waves is up-shifted by $80 \mathrm{MHz}$ we measure a fractional frequency instability of frequency division by 3 of better than $7.6 \times 10^{-14}$ for a measurement time of 10 $\mathrm{s}$, which is limited by the resolution of the rf spectrum analyzer used. Again, this agrees well with the theoretical prediction.

In conclusion, the experimental results of the current work prove the successful operation of an all-optical divider by 3 , whose properties are in good agreement with theory. Together with the observed high precision of the divider and its wavelength flexibility (due to the use of wavelengthtunable diode lasers and quasi-phase-matching in periodically poled crystals), self-phase-locked OPOs might show a considerable potential for future precision optical metrology.

\section{ACKNOWLEDGMENTS}

The authors acknowledge financial support by the Deutsche Forschungsgemeinschaft (DFG) and by the Dutch Stichting Voor Fundamenteel Onderzoek der Materie (FOM). 
[1] N. C. Wong, Opt. Lett. 15, 1129 (1990).

[2] S. A. Diddams, D. J. Jones, J. Ye, S. T. Cundiff, J. L. Hall, J. K. Ranka, R. S. Windeler, R. Holzwarth, Th. Udem, and T. W. Hänsch, Phys. Rev. Lett. 84, 5102 (2000).

[3] R. Graham and H. Haken, Z. Phys. 210, 276 (1968).

[4] D. Lee and N. C. Wong, Opt. Lett. 17, 13 (1992).

[5] S. Slyusarev, T. Ikegami, and S. Ohshima, Opt. Lett. 24, 1856 (1999).

[6] A. Douillet, J.-J. Zondy, G. Santarelli, A. Makdissi, and A. Clairon, IEEE Trans. Instrum. Meas. 50, 548 (2001).

[7] A. E. Siegman, Lasers (University Science Books, Mill Valley, CA, 1986), Chap. 29, p. 1130.

[8] C. D. Nabors, S. T. Yang, T. Day, and R. L. Byer, J. Opt. Soc. Am. B 7, 815 (1990).

[9] E. J. Mason and N. C. Wong, Opt. Lett. 23, 1733 (1998).

[10] D.-H. Lee, M. E. Klein, J.-P. Meyn, P. Groß, R. Wallenstein, and K.-J. Boller, Opt. Express 5, 114 (1999).

[11] D.-H. Lee, M. E. Klein, J.-P. Meyn, P. Groß, S. Marzenell, R. Wallenstein, and K.-J. Boller, Proc. SPIE 3928, 25 (2000).

[12] J.-J. Zondy, A. Douillet, A. Tallet, E. Ressayre, and M. Le Berre, Phys. Rev. A 63, 023814 (2001).
[13] W. Brunner and H. Paul, Opt. Commun. 19, 253 (1976).

[14] C. Fabre, P. F. Cohadon, and C. Schwob, Quantum Semiclassic. Opt. 9, 165 (1997).

[15] C. Fabre, E. J. Mason, and N. C. Wong, Opt. Commun. 170, 299 (1999).

[16] D.-H. Lee, M. E. Klein, and K.-J. Boller, Appl. Phys. B: Lasers Opt. 66, 747 (1998).

[17] K. P. Chung and A. Marcano O., J. Opt. Soc. Am. B 5, 2524 (1988).

[18] M. Scheidt, B. Beier, K.-J. Boller, and R. Wallenstein, Opt. Lett. 22, 1287 (1997).

[19] R. W. P. Drever, J. L. Hall, F. V. Kowalski, J. Hough, G. M. Ford, A. J. Munley, and H. Ward, Appl. Phys. B: Photophys. Laser Chem. 31, 97 (1983).

[20] P. L. Hansen and P. Buchhave, Opt. Lett. 22, 1074 (1997).

[21] D.-H. Lee, P. Groß, M. E. Klein, J.-P. Meyn, R. Wallenstein, and K.-J. Boller, in Proceedings of the Conference on Lasers and Electro-optics, San Francisco, 2000, OSA Technical Digest (Optical Society of America, Washington, DC, 2000), p. 523. 